TRANSACTIONS OF THE

AMERICAN MATHEMATICAL SOCIETY

Volume 351, Number 3, March 1999, Pages 1063-1074

S 0002-9947(99)02013-9

\title{
ON THE DIOPHANTINE EQUATION
}

$$
\left(x^{3}-1\right) /(x-1)=\left(y^{n}-1\right) /(y-1)
$$

MAOHUA LE

\begin{abstract}
In this paper we prove that the equation $\left(x^{3}-1\right) /(x-1)=$ $\left(y^{n}-1\right) /(y-1), x, y, n \in \mathbb{N}, x>1, y>1, n>3$, has only the solutions $(x, y, n)=(5,2,5)$ and $(90,2,13)$ with $y$ is a prime power. The proof depends on some new results concerning the upper bounds for the number of solutions of the generalized Ramanujan-Nagell equations.
\end{abstract}

\section{INTRODUCTION}

Let $\mathbb{Z}, \mathbb{N}, \mathbb{Q}$ be the sets of integers, positive integers and rational numbers respectively. For any positive integer $N$ with $N>2$, let $s(N)$ denote the number of solutions $(x, m)$ of the equation

$$
N=\frac{x^{m}-1}{x-1}, \quad x, m \in \mathbb{N}, \quad x>1, \quad m>2 .
$$

Eightly years ago, Ratat [13] and Goormaghtigh [4] observed that $s(31)=2$ and $s(8191)=2$, respectively. Simultaneously, they conjectured that if $N \notin\{31,8191\}$, then $s(N) \leq 1$. The problem can be written in the following form

Conjecture A. The equation

$$
\frac{x^{m}-1}{x-1}=\frac{y^{n}-1}{y-1}, \quad x, y, m, n \in \mathbb{N}, \quad x>1, \quad y>1, \quad n>m>2,
$$

has only the solutions $(x, y, m, n)=(5,2,3,5)$ and $(90,2,3,13)$.

In addition, Bateman (see [5, Problem B25]) asked the following problem.

Problem B. Is $(x, y, m, n)=(5,2,3,5)$ the only solution of (2) for which $x, y$ and $\left(x^{m}-1\right) /(x-1)$ are all prime powers?

These are two rather difficult questions. By the results of Baker, Davenport, Lewis, Schinzel, Shorey and Tijdeman (see the references of [14]), we see that (2) has only finitely many solutions $(x, y, m, n)$ if any two out of the four variables $x, y, m$ and $n$ are fixed. However, to this day we do not know whether (2) has only finitely many solutions $(x, y, m, n)$ if any one out of $x, y, m$ and $n$ is fixed.

In this paper we discuss the finiteness of solutions of (2) by the means of the generalized Ramanujan-Nagell equations. Let $D_{1}, D_{2}$ be coprime positive integers,

Received by the editors February 28, 1996 and, in revised form, September 18, 1996 and January 22, 1997.

1991 Mathematics Subject Classification. Primary 11D61, 11J86.

Supported by the National Natural Science Foundation of China and the Guangdong Provincial Natural Science Foundation.

(C)1999 American Mathematical Society 
and let $p$ be a prime with $p \nmid D_{1} D_{2}$. Further let $N\left(D_{1}, D_{2}, p\right)$ and $N^{\prime}\left(D_{1}, D_{2}, p\right)$ denote the numbers of the solutions $(r, s)$ and $\left(r^{\prime}, s^{\prime}\right)$ of equations

$$
D_{1} r^{2}+D_{2}=p^{s}, \quad r, s \in \mathbb{N},
$$

and

$$
D_{1} r^{2}+D_{2}=2 p^{s^{\prime}}, \quad r^{\prime}, s^{\prime} \in \mathbb{N},
$$

respectively. In this paper we prove the following two general results.

Theorem 1. $N\left(D_{1}, D_{2}, p\right) \leq 2$ except for $N(1,7,2)=5$ and $N(3,5,2)=3$.

Theorem 2. If $2 \nmid D_{1} D_{2}$ and $p$ is an odd prime, then $N^{\prime}\left(D_{1}, D_{2}, p\right) \leq 2$.

On applying the above-mentioned theorems to the equation (2), we shall deduce the following result.

Corollary. The equation (2) has only the solutions $(x, y, m, n)=(5,2,3,5)$ and $(90,2,3,13)$ such that $m=3$ and $y$ is a prime power.

\section{Proof of Theorem 1}

First we may assume that $D_{1}$ is squarefree. In [1] and [2], Apéry proved that $N\left(1, D_{2}, p\right) \leq 2$ except for $N(1,7,2)=5$. In [7] and [8], the author proved that $N\left(D_{1}, 1, p\right) \leq 2$ and $N\left(D_{1}, D_{2}, 2\right) \leq 2$ except for $N(1,7,2)=5$ and $N(3,5,2)=3$, respectively. Therefore, we may assume that $D_{1}>1, D_{2}>1$ and $p$ is an odd prime.

Lemma 1 ([10, Formula 3.76]). For any positive integer $t$ and any complex numbers $\alpha$ and $\beta$, we have

$$
\alpha^{t}+\beta^{t}=\sum_{i=0}^{[t / 2]}(-1)^{i}\left[\begin{array}{l}
t \\
i
\end{array}\right](\alpha+\beta)^{t-2 i}(\alpha \beta)^{i},
$$

where $[t / 2]$ is the largest integer which does not exceed $t / 2$,

$$
\left[\begin{array}{l}
t \\
i
\end{array}\right]=\frac{(t-i-1) ! t}{(t-2 i) ! i !}, \quad i=0,1, \ldots,\left[\frac{t}{2}\right],
$$

are positive integers.

Lemma 2 ([9, Lemma 4$])$. If the equation

$$
D_{1} X^{2}+D_{2} Y^{2}=p^{z}, \quad X, Y, Z \in \mathbb{Z}, \quad \operatorname{gcd}(X, Y)=1, \quad Z>0,
$$

has solutions $(X, Y, Z)$, then it has a unique solution $\left(X_{1}, Y_{1}, Z_{1}\right)$ such that $X_{1}>$ $0, Y_{1}>0$ and $Z_{1} \leq Z$, where $Z$ runs through all solutions $(X, Y, Z)$ of $(5)$. $\left(X_{1}, Y_{1}, Z_{1}\right)$ is called the least solution of (5). Further, every solution $(X, Y, Z)$ of (5) can be expressed as

$$
\begin{aligned}
Z=Z_{1} t, X \sqrt{D_{1}}+Y \sqrt{-D_{2}}=\lambda_{1}\left(X_{1} \sqrt{D_{1}}+\lambda_{2} Y_{1} \sqrt{-D_{2}}\right)^{t}, & t \in \mathbb{N}, 2 \nmid t, \lambda_{1}, \lambda_{2} \in\{-1,1\} .
\end{aligned}
$$

Lemma 3. If $N\left(D_{1}, D_{2}, p\right)>2$, then (3) has two solutions $\left(r_{1}, s_{1}\right)$ and $\left(r_{2}, s_{2}\right)$ such that

$$
s_{1}=Z_{1} t_{1}, \quad s_{2}=Z_{1} t_{2}, \quad 1<t_{1}<t_{2},
$$


where $\left(X_{1}, Y_{1}, Z_{1}\right)$ is the least solution of (5), $t_{1}$ and $t_{2}$ are odd primes satisfying

$$
\begin{gathered}
\sum_{i=0}^{\left(t_{j}-1\right) / 2}\left[\begin{array}{c}
t_{j} \\
i
\end{array}\right]\left(-4 D_{2}\right)^{\left(t_{j}-1\right) / 2-i} p^{Z_{1} i}=(-1)^{\left(t_{j}-1\right)\left(p^{Z_{1}}\right) / 4}, \quad j=1,2, \\
\frac{\pi}{2 \arcsin \left(D_{2} / p^{s_{1}}\right)^{1 / 2}}<t_{2}
\end{gathered}
$$

and

$$
\left|\arcsin \left(\frac{D_{2}}{p^{Z_{1}}}\right)^{1 / 2}-\frac{k \pi}{t_{j}}\right|<\frac{\pi}{2 t_{j} p^{Z_{1}\left(t_{j}-1\right) / 2}}, \quad j=1,2,
$$

where $k$ is a positive integer satisfying $k \leq\left(t_{j}-1\right) / 2$.

Proof. We see that if (3) has a solution $(r, s)$, then (5) has a solution $(X, Y, Z)=$ $(r, 1, s)$. It follows from Lemma 2 that the least solution $\left(X_{1}, Y_{1}, Z_{1}\right)$ of $(5)$ satisfies $Y_{1}=1$. Then $(r, s)=\left(X_{1}, Z_{1}\right)$ is a solution of (3). Further, let

$$
\varepsilon=X_{1} \sqrt{D_{1}}+\sqrt{-D_{2}}, \quad \bar{\varepsilon}=X_{1} \sqrt{D_{1}}-\sqrt{-D_{2}} .
$$

By the proof of [9, Theorem 1], if $(r, s)$ is a solution of $(3)$ with $(r, s) \neq\left(X_{1}, Z_{1}\right)$, then there exists a positive integer $t$ satisfying $t>1,2 \nmid t$,

$$
s=Z_{1} t
$$

and

$$
\left|\frac{\varepsilon^{t}-\bar{\varepsilon}^{t}}{\varepsilon-\bar{\varepsilon}}\right|=1
$$

Therefore, by (11), if $N\left(D_{1}, D_{2}, p\right)>2$, then (3) has two solutions $\left(r_{1}, s_{1}\right)$ and $\left(r_{2}, s_{2}\right)$ satisfying (6). Moreover, we may assume that (3) has no solutions $(r, s)$ satisfying

$$
Z_{1}<s<s_{1}, \quad s_{1}<s<s_{2} .
$$

Since $\varepsilon-\bar{\varepsilon}=2 \sqrt{-D_{2}}$ and $\varepsilon \bar{\varepsilon}=p^{Z_{1}}$, by Lemma 1 , we get from (12) that

$$
\begin{aligned}
\frac{\varepsilon^{t}-\bar{\varepsilon}^{t}}{\varepsilon-\bar{\varepsilon}} & =\sum_{i=0}^{(t-1) / 2}(-1)^{i}\left[\begin{array}{l}
t \\
i
\end{array}\right](\varepsilon-\bar{\varepsilon})^{t-2 i-1}(-\varepsilon \bar{\varepsilon})^{i}=\sum_{i=0}^{(t-1) / 2}\left[\begin{array}{l}
t \\
i
\end{array}\right]\left(-4 D_{2}\right)^{(t-1) / 2-i} p^{Z_{1}} \\
& = \pm 1
\end{aligned}
$$

Since $t=(-1)^{(t-1) / 2}(\bmod 4)$ and $p^{Z_{1}} \equiv(-1)^{\left(p^{Z_{1}}-1\right) / 2}(\bmod 4)$, we obtain $(7)$ from (14).

For any positive integer $t$ with $2 \nmid t$, let

$$
X_{t}=\left|\frac{\varepsilon^{t}+\bar{\varepsilon}^{t}}{2 \sqrt{D_{1}}}\right|, \quad Y_{t}=\left|\frac{\varepsilon^{t}-\bar{\varepsilon}^{t}}{\varepsilon-\bar{\varepsilon}}\right| .
$$

By Lemma 1, $X_{t}$ and $Y_{t}$ are positive integers satisfying

$$
D_{1} X_{t}^{2}+D_{2} Y_{t}^{2}=p^{Z_{1} t} .
$$

Further, by Lemma 2, we see from (16) that $(r, s)$ is a solution of (3) satisfying (11) if and only if $Y_{t}=1$. From (7), we get

$$
Y_{t_{1}}=Y_{t_{2}}=1 \text {. }
$$


If $t_{1}$ is not an odd prime, then $t_{1}=k_{1} k_{2}$, where $k_{1}$ and $k_{2}$ are positive integers satisfying $k_{1}>1, k_{2}>1$ and $2 \nmid k_{1} k_{2}$. On applying Lemma 1 , we find from (17) that

$$
1=Y_{t_{1}}=Y_{k_{1}}\left|\frac{\left(\varepsilon^{k_{1}}\right)^{k_{2}}-\left(\bar{\varepsilon}^{k_{1}}\right)^{k_{2}}}{\varepsilon^{k_{1}}-\bar{\varepsilon}^{k_{1}}}\right|=Y_{k_{1}}\left|\sum_{i=0}^{\left(k_{2}-1\right) / 2}\left[\begin{array}{c}
k_{2} \\
i
\end{array}\right]\left(-4 D_{2} Y_{k_{1}}^{2}\right)^{\left(k_{2}-1\right) / 2-i} p^{Z_{1} k_{1} i}\right| .
$$

Hence, we get from (18) that $Y_{k_{1}}=Y_{t_{1}} / Y_{k_{1}}=1$. It implies that (3) has a solution $(r, s)=\left(X_{k_{1}}, Z_{1} k_{1}\right)$ satisfying $Z_{1}<s<s_{1}$, which contradicts (13). Thus $t_{1}$ must be an odd prime.

By the same argument, if $t_{2}$ is not an odd prime, then from (13) we get $t_{2}=t_{1}^{2}$ and $Y_{t_{2}} / Y_{t_{1}}=1$. In this case, by Lemma 1, we have

$$
\frac{Y_{t_{2}}}{Y_{t_{1}}}=\left|\frac{\varepsilon^{t_{1}^{2}}-\bar{\varepsilon}^{t_{1}^{2}}}{\varepsilon^{t_{1}}-\bar{\varepsilon}^{t_{1}}}\right|=\left|\sum_{i=0}^{\left(t_{1}-1\right) / 2}\left[\begin{array}{c}
t_{1} \\
i
\end{array}\right]\left(-4 D_{2}\right)^{\left(t_{1}-1\right) / 2-i} p^{Z_{1} t_{1} i}\right|=1 .
$$

Since $(-1)^{\left(t_{1}-1\right)\left(p^{z_{1}}+1\right) / 4}=(-1)^{\left(t_{1}-1\right)\left(p^{z_{1} t_{1}}+1\right) / 4}$, we get from (19) that

$$
\sum_{i=0}^{\left(t_{1}-1\right) / 2}\left[\begin{array}{c}
t_{1} \\
i
\end{array}\right]\left(-4 D_{2}\right)^{\left(t_{1}-1\right) / 2-i} p^{z_{1} t_{1} i}=(-1)^{\left(t_{1}-1\right)\left(p^{Z_{1}}+1\right) / 4} .
$$

Further, since $t_{1} \geq 3$, we see from (20) that

$$
\left(-4 D_{2}\right)^{\left(t_{1}-1\right) / 2}-(-1)^{\left(t_{1}-1\right)\left(p^{Z_{1}}+1\right) / 4} \equiv 0\left(\bmod p^{3 Z_{1}}\right) .
$$

On the other hand, by (7), we have

$$
\begin{gathered}
\left(\left(-4 D_{2}\right)^{\left(t_{1}-1\right) / 2}-(-1)^{\left(t_{1}-1\right)\left(p^{Z_{1}}+1\right) / 4}\right)+\left[\begin{array}{c}
t_{1} \\
1
\end{array}\right]\left(-4 D_{2}\right)^{\left(t_{1}-3\right) / 2} p^{Z_{1}} \\
+\left[\begin{array}{c}
t_{1} \\
2
\end{array}\right]\left(-4 D_{2}\right)^{\left(t_{1}-5\right) / 2} p^{2 Z_{1}} \equiv 0 \quad\left(\bmod p^{3 Z_{1}}\right) .
\end{gathered}
$$

The combination of (21) and (22) yields

$$
t_{1}\left(4 D_{2}-\left(\frac{t_{1}-3}{2}\right) p^{Z_{1}}\right) \equiv 0 \quad\left(\bmod p^{2 Z_{1}}\right) .
$$

Since $p \nmid D_{2}$ and $t_{1}$ is an odd prime, (23) is impossible. Thus $t_{2}$ is an odd prime too.

By (10), we have

$$
\varepsilon=p^{Z_{1} / 2} e^{\theta \sqrt{-1}}, \quad \bar{\varepsilon}=p^{Z_{1} / 2} e^{-\theta / \sqrt{-1}},
$$

where $\theta$ is a real number satisfying

$$
\sin \theta=\frac{\varepsilon-\bar{\varepsilon}}{2 p^{Z_{1} / 2} \sqrt{-1}}=\left(\frac{D_{2}}{p^{Z_{1}}}\right)^{1 / 2} .
$$

Since $0<D_{2} / p^{Z_{1}}<1$, we may assume that $\theta$ satisfies

$$
0<\theta<\frac{\pi}{2}
$$

Further, sine $Y_{t_{1}}=Y_{t_{2}}=1$ by (17), we get from (24) and (25) that

$$
\sin t_{j} \theta=(-1)^{\left(t_{j}-1\right)\left(p^{Z_{1}}+1\right) / 4} \frac{\sin \theta}{p^{Z_{1}\left(t_{j}-1\right) / 2}}, \quad j=1,2 .
$$


Hence

$$
t_{j} \theta=k \pi+(-1)^{k+\left(t_{j}-1\right)\left(p^{Z_{1}}+1\right) / 4} \phi_{j}, \quad j=1,2,
$$

where $k$ is an integer, $\phi_{j}(j=1,2)$ are positive numbers satisfying

$$
\sin \phi_{j}=\frac{\sin \theta}{p^{Z_{1}\left(t_{j}-1\right) / 2}}, \quad 0<\phi_{j}<\frac{\pi}{2}, \quad j=1,2 .
$$

Notice that $0<\theta<\pi / 2$ by (26). Since $0<\phi_{j}<\theta<\pi / 2 \quad(j=1,2)$ by (26) and (29), we see from (28) that $k$ satisfies $1 \leq k \leq\left(t_{j}-1\right) / 2$. Thus, by (25), (28) and (29), we get

$$
\begin{aligned}
\left|\arcsin \left(\frac{D_{2}}{p^{Z_{1}}}\right)^{1 / 2}-\frac{k \pi}{t_{j}}\right|=\left|\theta-\frac{k \pi}{t_{j}}\right|=\frac{\phi_{j}}{t_{j}}=\frac{\arcsin \left((\sin \theta) / p^{Z_{1}\left(t_{j}-1\right) / 2}\right)}{t_{j}} \\
<\frac{\arcsin \left(p^{-Z_{1}\left(t_{j}-1\right) / 2}\right.}{t_{j}}<\frac{\pi}{2 t_{j} p^{Z_{1}\left(t_{j}-1\right) / 2}}, \quad j=1,2 .
\end{aligned}
$$

The inequality (9) is proved.

From (25) and (27), we get

$$
\left|\sin t_{1} \theta\right|=\left(\frac{D_{2}}{p^{s_{1}}}\right)^{1 / 2}, \quad\left|\sin t_{2} \theta\right|=\left(\frac{D_{2}}{p^{s_{2}}}\right)^{1 / 2} .
$$

By (30), there exist suitable nonnegative integers $k_{1}$ and $k_{2}$ such that

$$
\left|k_{1} \pi-t_{1} \theta\right|=\arcsin \left(\frac{D_{2}}{p^{s_{1}}}\right)^{1 / 2}, \quad\left|k_{2} \pi-t_{2} \theta\right|=\arcsin \left(\frac{D_{2}}{p^{s_{2}}}\right)^{1 / 2} .
$$

Since $s_{1}<s_{2}$ and $\arcsin \left(D_{2} / p^{s_{1}}\right)^{1 / 2}>\arcsin \left(D_{2} / p^{s_{2}}\right)^{1 / 2}$, we get from (31) that

$0<\left|\frac{k_{1}}{t_{1}}-\frac{k_{2}}{t_{2}}\right| \pi \leq \frac{1}{t_{1}} \arcsin \left(\frac{D_{2}}{p^{s_{1}}}\right)^{1 / 2}+\frac{1}{t_{2}} \arcsin \left(\frac{D_{2}}{p^{s_{2}}}\right)^{1 / 2}<\frac{2}{t_{1}} \arcsin \left(\frac{D_{2}}{p^{s_{1}}}\right)^{1 / 2}$.

Notice that $\left|k_{1} / t_{1}-k_{2} / t_{2}\right| \geq 1 / t_{1} t_{2}$ if $k_{1} / t_{1} \neq k_{2} / t_{2}$. We obtain (8) from (32). The lemma is proved.

Lemma 4. If (3) has two solutions $\left(r_{1}, s_{1}\right)$ and $\left(r_{2}, s_{2}\right)$ satisfying $(6)$ with $t_{1}=3$, then we have

$$
4 D_{2}=3 p^{Z_{1}}+(-1)^{\left(p^{Z_{1}-1}\right) / 2}
$$

and

$$
t_{2} \geq 2 p^{2 Z_{1}}+3
$$

Proof. Since $t_{1}=3$, we get (33) from (7). Let $\delta=(-1)^{\left(p^{Z_{1}}+1\right) / 2}$. We get from (17) and (33) that

$$
\left(-3 p^{Z_{1}}+\delta\right)^{\left(t_{2}-1\right) / 2}+t_{2}\left(-3 p^{Z_{1}}+\delta\right)^{\left(t_{2}-3\right) / 2}\left(3 p^{Z_{1}}\right) \equiv \delta^{\left(t_{2}-1\right) / 2} \quad\left(\bmod p^{2 Z_{1}}\right),
$$

whence we obtain $\left(t_{2}-3\right) / 2 \equiv 0\left(\bmod p^{Z_{1}}\right)$. By $(7)$ again, we get

$$
-\left(\frac{t_{2}-3}{2}\right)+\left(9\left(\begin{array}{c}
\left(t_{2}-1\right) / 2 \\
2
\end{array}\right)-3\left[\begin{array}{c}
t_{2} \\
1
\end{array}\right]\left(\begin{array}{c}
\left(t_{2}-3\right) / 2 \\
1
\end{array}\right)+\left[\begin{array}{c}
t_{2} \\
2
\end{array}\right]\right) \delta p^{Z_{1}} \equiv 0\left(\bmod p^{2 Z_{1}}\right) .
$$


Notice that

$$
\left(\begin{array}{c}
\left(t_{2}-1\right) / 2 \\
2
\end{array}\right) \equiv\left(\begin{array}{c}
\left(t_{2}-3\right) / 2 \\
1
\end{array}\right) \equiv\left[\begin{array}{c}
t_{2} \\
2
\end{array}\right] \equiv 0 \quad\left(\bmod \frac{t_{2}-3}{2 \lambda}\right),
$$

where $\lambda=1$ or 2 according to whether $t_{2} \equiv 1(\bmod 4)$ or not. We find from $(35)$ that $\left(t_{2}-3\right) / 2 \equiv 0\left(\bmod p^{2 Z_{1}}\right)$. Since $t_{2}>3$, it implies (34). The lemma is proved.

Let $\alpha$ be an algebraic number of degree $d$ with conjugates $\sigma_{1} \alpha, \sigma_{2} \alpha, \ldots, \alpha_{d} \alpha$ and the minimal polynomial

$$
a_{0} z^{d}+a_{1} z^{d-1}+\cdots+a_{d}=a_{0} \prod_{i=1}^{d}\left(z-\sigma_{i} \alpha\right) \in \mathbb{Z}[z], \quad a_{0}>0 .
$$

Then

$$
h(\alpha)=\frac{1}{d}\left(\log a_{0}+\sum_{i=1}^{d} \log \max \left(1,\left|\sigma_{i} \alpha\right|\right)\right)
$$

is called the logarithmic absolute height of $\alpha$.

Lemma 5 ([6, Théorème 3$])$. Let $\alpha$ be an algebraic number with $|\alpha|=1$. Let $b_{1}, b_{2}$ be positive integers, and let $\Lambda=b_{1} \log \alpha-b_{2} \pi \sqrt{-1}$. If $\alpha$ is not a root of unity, then we have

$$
\log |\Lambda| \geq-8.87 A B^{2}
$$

where $A=\max (20,10.98|\log \alpha|+r h(\alpha)), B=\max \left(17, r^{1 / 2 / 10}, 5.03+2.35 r+\right.$ $\left.r \log \left(b_{1} / 68.9+b_{2} / 2 A\right)\right), r=[\mathbb{Q}(\alpha): \mathbb{Q}] / 2$.

Lemma 6. If (3) has two solutions $\left(r_{1}, s_{1}\right)$ and $\left(r_{2}, s_{2}\right)$ satisfying (6), then we have

$$
t_{2}<2+2563.43\left(1+\frac{10.98 \pi}{\log p^{Z_{1} / 2}}\right) .
$$

Proof. Under the assumption, we get from (15) and (17) that

$$
2 p^{Z_{1} / 2}>2 D_{2}^{1 / 2}|\varepsilon-\bar{\varepsilon}|=\left|\varepsilon^{t_{2}}-\bar{\varepsilon}^{t_{2}}\right| .
$$

Let $\alpha=\varepsilon / \bar{\varepsilon}$. Then from (37) we get

$$
\log 2 p^{Z_{1} / 2}>\log \left|\bar{\varepsilon}^{t_{2}}\right|+\log \left|\alpha^{t_{2}}-1\right|=t_{2} \log p^{Z_{1} / 2}+\log \left|\alpha^{t_{2}}-1\right| .
$$

Since $t_{2} \geq 5$, if $\left|\alpha^{t_{2}}-1\right| \geq 1$, then (38) is impossible. Therefore, we have

$$
\left|\alpha^{t_{2}}-1\right| \geq\left|t_{2} \log \alpha-k \pi \sqrt{-1}\right|
$$

for some positive integers $k$ with $k \leq t_{2}$. Let $\Lambda=t_{2} \log \alpha-k \pi \sqrt{-1}$. From (38) and (39), we get

$$
\log 2 p^{Z_{1} / 2}-\log |\Lambda|>t_{2} \log p^{Z_{1} / 2} .
$$

We see from (10) that $\alpha$ satisfies

$$
p^{Z_{1}} \alpha^{2}-2\left(D_{1} X_{1}^{2}-D_{2}\right) \alpha+p^{Z_{1}}=0 .
$$


It implies that $[\mathbb{Q}(\alpha): \mathbb{Q}]=2, h(\alpha)=\log p^{Z_{1} / 2}$ and $\alpha$ is not a root of unity. Further, by (24) and (26), we have $|\alpha|=1$ and $|\log \alpha|=2 \theta<\pi$. Since $k \leq t_{2}$, by Lemma 5, we get

$$
\begin{aligned}
\log |\Lambda| \geq & -8.87\left(10.98 \pi+\log p^{Z_{1} / 2}\right) \\
& \times\left(\max \left(17,7.38+\log \left(\frac{t_{2}}{68.9}+\frac{t_{2}}{2\left(10.98 \pi+\log p^{Z_{1} / 2}\right)}\right)\right)\right)^{2} .
\end{aligned}
$$

If

$$
7.38+\log \left(\frac{t_{2}}{68.9}+\frac{t_{2}}{2\left(10.98 \pi+\log p^{Z_{1} / 2}\right)}\right) \geq 17
$$

then we have

$$
\log 0.0288015 t_{2} \geq 9.62
$$

whence we conclude that

$$
t_{2}>523063
$$

On the other hand, by (40), (41) and (42), we get

$$
\begin{gathered}
3+565.8842\left(7.38+\log 0.0288015 t_{2}\right)^{2}>1+\frac{\log 2}{\log p^{Z_{1} / 2}}+8.87\left(1+\frac{10.98 \pi}{\log p^{Z_{1} / 2}}\right) \\
\left(7.38+\log \left(\frac{t_{2}}{68.9}+\frac{t_{2}}{2\left(10.98 \pi+\log p^{Z_{1} / 2}\right.}\right)\right)^{2}>t_{2} .
\end{gathered}
$$

We calculate from (44) that $t_{2}<150000$. It contradicts with (43). So we have

$$
7.38+\log \left(\frac{t_{2}}{68.9}+\frac{t_{2}}{2\left(10.98 \pi+\log p^{Z_{1} / 2}\right)}\right)<17 .
$$

Thus, by (40), (41) and (45), we obtain (36). The lemma is proved.

Lemma 7 ([3]). The equation

$$
X^{3}+X^{2} Y-2 X Y^{2}-Y^{3}=1, \quad X, Y \in \mathbb{Z}
$$

has only the solutions $(X, Y)=(1,0),(0,-1),(-1,1),(2,-1),(-1,2),(5,4),(4,-9)$ and $(-9,5)$.

Proof of Theorem 1. We now suppose that $N\left(D_{1}, D_{2}, p\right)>2$. By Lemma 3 , then (3) has two solutions $\left(r_{1}, s_{1}\right)$ and $\left(r_{2}, s_{2}\right)$ satisfying $(6)$.

By Lemmas 4 and 6 , if $t_{1}=3$, then we have

$$
2 p^{2 Z_{1}}+3 \leq t_{2}<2+2563.43\left(1+\frac{10.98 \pi}{\log p^{Z_{1} / 2}}\right),
$$

whence we conclude that $p^{Z_{1}} \leq 137$. Recall that $D_{1}>1, D_{2}>1$ and $D_{1}$ is square free. By (33), we only need to consider the cases $\left(D_{1}, D_{2}, p^{Z_{1}}\right)=(2,5,7),(2,7,9)$, $(3,8,11),(3,10,13),(5,14,19),(6,17,23),(6,19,25),(7,20,27),(7,22,29),(2,23,31)$, $(10,31,41), \quad(11,32,43), \quad(3,35,47), \quad(3,37,49), \quad(13,40,53), \quad(15,44,59), \quad(15,46,61)$, $(17,50,67),(2,53,71),(2,55,73),(5,59,79),(5,61,81),(21,62,83),(22,67,89),(6,73,97)$, 
$(26,77,103),(3,80,107),(3,82,109),(7,85,113),(30,91,121),(31,94,125),(2,95,127)$, $(33,98,131)$ and $(34,103,137)$. On the other hand, by $(9)$ and $(33)$, we get

$$
\left|\arcsin \left(\frac{3}{4}+\frac{(-1)^{\left(p^{Z_{1}}-1\right) / 2}}{4 p^{Z_{1}}}\right)^{1 / 2}-\frac{k \pi}{t_{2}}\right|<\frac{\pi}{2 t_{2} p^{Z_{1}\left(t_{2}-1\right) / 2}}
$$

for some positive integers $k$ with $k \leq\left(t_{2}-1\right) / 2$. However, by (46), (47) is false for the above-mentioned cases.

If $t_{1}=5$, then from (7) we get

$$
16 D_{2}^{2}-20 D_{2} p^{Z_{1}}+5 p^{2 Z_{1}}=1 .
$$

Since $p^{Z_{1}}$ is an odd prime power, we see from $(48)$ that $4 D_{2} \equiv \lambda\left(\bmod p^{Z_{1}}\right)$, where $\lambda \in\{-1,1\}$. So we have $4 D_{2}=k p^{Z_{1}}+\lambda$, where $k=1$ or 3 . Hence, by (48), we get

$$
p^{Z_{1}}\left(k^{2}-5 k+5\right)=(5-2 k) \lambda .
$$

This implies that $p^{Z_{1}}=3$. Since $D_{2}$ is an integer with $D_{2}>1$, this is impossible.

If $t_{1}=7$, then we have

$$
\left(2 p^{Z_{1}}-4 D_{2}\right)^{3}+\left(2 p^{Z_{1}}-4 D_{2}\right)^{2} p^{Z_{1}}-2\left(2 p^{Z_{1}}-4 D_{2}\right) p^{2 Z_{1}}-p^{3 Z_{1}}= \pm 1
$$

by (7). However, by Lemma 7, (49) is impossible.

From (8) and (36), if $t_{1}>7$, then we have $t_{1} \geq 11$ and

$$
\begin{aligned}
p^{5 Z_{1}} & \leq p^{Z_{1}\left(t_{1}-1\right) / 2}<\left(\frac{p^{Z_{1} t_{1}}}{D_{2}}\right)^{1 / 2}<\frac{\pi}{2 \arcsin \left(D_{2} / p^{Z_{1} t_{1}}\right)^{1 / 2}}<t_{2} \\
& <2+2563.42\left(1+\frac{10.98 \pi}{\log p^{Z_{1} / 2}}\right),
\end{aligned}
$$

whence we calculate that $p^{Z_{1}} \leq 9$. Since, if $\left(D_{1}, D_{2}, p^{Z_{1}}\right)=(2,5,7)$ or $(2,7,9)$, then $t_{1}=3$. So we only need to consider the cases $\left(D_{1}, D_{2}, p^{Z_{1}}\right)=(2,3,5),(3,2,5)$, $(3,4,7),(5,2,7),(5,4,9)$ and $(7,2,9)$. For the above-mentioned cases, $(7)$ is false if $t_{1}=11$ or 13 . So we have $t_{1} \geq 17$. Then, by (8) and (36) again, we get

$$
390625 \leq p^{8 Z_{1}} \leq p^{Z_{1}\left(t_{1}-1\right) / 2}<t_{2}<2+2563.42\left(1+\frac{10.98 \pi}{\log 5^{1 / 2}}\right)<112451,
$$

a contradiction. All cases have been considered, the proof is complete.

\section{Proof of Theorem 2}

By the same method as in the proofs of Lemmas 2, 3, 4 and 6 , we can prove the corresponding lemmas about the equation (4) without any difficulty.

Lemma 8. If the equation

$$
D_{1} X^{\prime 2}+D_{2} Y^{\prime 2}=2 p^{Z^{\prime}}, \quad X^{\prime}, Y^{\prime}, Z^{\prime} \in \mathbb{Z}, \quad \operatorname{gcd}\left(X^{\prime}, Y^{\prime}\right)=1, \quad Z^{\prime}>0,
$$

has solutions $\left(X^{\prime}, Y^{\prime}, Z^{\prime}\right)$, then it has a unique solution $\left(X_{1}^{\prime}, Y_{1}^{\prime}, Z_{1}^{\prime}\right)$ such that $X_{1}^{\prime}>0, Y_{1}^{\prime}>0$ and $Z_{1}^{\prime} \leq Z^{\prime}$, where $Z^{\prime}$ runs through all solutions $\left(X^{\prime}, Y^{\prime}, Z^{\prime}\right)$ of $(50)$. $\left(X_{1}^{\prime}, Y_{1}^{\prime}, Z_{1}^{\prime}\right)$ is called the least solution of (50). Moreover, every solution $\left(X^{\prime}, Y^{\prime}, Z^{\prime}\right)$ of (50) can be expressed as

$$
\begin{aligned}
Z^{\prime}=Z_{1}^{\prime} t^{\prime}, \frac{X^{\prime} \sqrt{D_{1}}+Y^{\prime} \sqrt{-D_{2}}}{\sqrt{2}}=\lambda_{1}\left(\frac{X_{1}^{\prime} \sqrt{D_{1}}+\lambda_{2} Y_{1}^{\prime} \sqrt{-D_{2}}}{\sqrt{2}}\right)^{t^{0}}, \\
t^{\prime} \in \mathbb{N}, 2 \nmid t^{\prime}, \lambda_{1}, \lambda_{2} \in\{-1,1\} .
\end{aligned}
$$


Lemma 9. The equation (4) has solutions $\left(r^{\prime}, s^{\prime}\right)$ if and only if (50) has solutions $\left(X^{\prime}, Y^{\prime}, Z^{\prime}\right)$ and its least solution $\left(X_{1}^{\prime}, Y_{1}^{\prime}, Z_{1}^{\prime}\right)$ satisfies $Y_{1}^{\prime}=1$. Moreover, if $N^{\prime}\left(D_{1}, D_{2}, p\right)>2$, then (4) has two solutions $\left(r_{1}^{\prime}, s_{1}^{\prime}\right)$ and $\left(r_{2}^{\prime}, s_{2}^{\prime}\right)$ such that

$$
s_{1}^{\prime}=Z_{1}^{\prime} t_{1}^{\prime}, \quad s_{2}^{\prime}=Z_{1}^{\prime} t_{2}^{\prime}, \quad 1<t_{1}^{\prime}<t_{2}^{\prime},
$$

where $t_{1}^{\prime}$ and $t_{2}^{\prime}$ are odd primes satisfying

$$
\begin{gathered}
\sum_{i=0}^{\left(t_{j}^{\prime}-1\right) / 2}\left[\begin{array}{c}
t_{j}^{\prime} \\
i
\end{array}\right]\left(-2 D_{2}\right)^{\left(t_{j}^{\prime}-1\right) / 2-i} p_{1}^{Z_{1}^{\prime}}=-(-1)^{\left(t_{j}^{\prime}-1\right)\left(p^{Z_{1}^{\prime}}+1\right) / 4}, \quad j=1,2, \\
\frac{\pi}{2 \arcsin \left(D_{2} / 2 p^{s_{1}^{\prime}}\right)^{1 / 2}}<t_{2}^{\prime}
\end{gathered}
$$

and

$$
\left|\arcsin \left(\frac{D_{2}}{2 p^{Z_{1}^{\prime}}}\right)^{1 / 2}-\frac{k \pi}{t_{j}^{\prime}}\right|<\frac{\pi}{2 t_{j}^{\prime} p^{Z_{1}^{\prime}\left(t_{j}^{\prime}-1\right) / 2}}, \quad j=1,2,
$$

where $k$ is a positive integer satisfying $k \leq\left(t_{j}^{\prime}-1\right) / 2$.

Lemma 10. If (4) has two solutions $\left(r_{1}^{\prime}, s_{1}^{\prime}\right)$ and $\left(r_{2}^{\prime}, s_{2}^{\prime}\right)$ satisfying (51) with $t_{1}^{\prime}=$ 3 , then we have

$$
2 D_{2}=3 p^{Z_{1}^{\prime}}+(-1)^{\left(p^{Z_{1}^{\prime}}+1\right) / 2}
$$

and

$$
t_{2}^{\prime} \geq 2 p^{2 Z_{1}^{\prime}}+3
$$

Lemma 11. If (4) has two solutions $\left(r_{1}^{\prime}, s_{1}^{\prime}\right)$ and $\left(r_{2}^{\prime}, s_{2}^{\prime}\right)$ satisfying (51), then we have

$$
t_{2}^{\prime}<3+2563.43\left(1+\frac{10.98 \pi}{\log p^{Z_{1}^{\prime} / 2}}\right) .
$$

Proof of Theorem 2. We now suppose that $N^{\prime}\left(D_{1}, D_{2}, p\right)>2$. By Lemma 9, then (4) has two solutions $\left(r_{1}^{\prime}, s_{1}^{\prime}\right)$ and $\left(r_{2}^{\prime}, s_{2}^{\prime}\right)$ satisfying (51).

By Lemmas 10 and 11, if $t_{1}^{\prime}=3$, then we have

$$
2 p^{2 Z_{1}^{\prime}}+3 \leq t_{2}^{\prime}<3+2563.43\left(1+\frac{10.98 \pi}{\log p^{Z_{1}^{\prime} / 2}}\right),
$$

whence we calculate that $p^{Z_{1}^{\prime}} \leq 137$. Hence, by (55), we only need to consider the cases $\left(D_{1}, D_{2}, p^{Z_{1}^{\prime}}\right)$ such that $p^{Z_{1}^{\prime}} \leq 137$ and $2 D_{2}=3 p^{Z_{1}^{\prime}}+(-1)^{\left(p_{1}^{Z_{1}^{\prime}}+1\right) / 2}$. On the other hand, by (54) and (55), we get

$$
\left|\arcsin \left(\frac{3}{4}+\frac{(-1)^{\left(p^{Z_{1}^{\prime}}+1\right) / 2}}{4 p^{Z_{1}^{\prime}}}\right)^{1 / 2}-\frac{k \pi}{t_{2}^{\prime}}\right|<\frac{\pi}{2 t_{2}^{\prime} p^{Z_{1}^{\prime}\left(t_{2}^{\prime}-1\right) / 2}}
$$

for some positive integers $k$ with $k \leq\left(t_{2}^{\prime}-1\right) / 2$. However, for the above-mentioned cases, if $t_{2}^{\prime}$ satisfies (58), then (59) is impossible.

If $t_{1}^{\prime}=5$, then from (52) we get

$$
4 D_{2}^{2}-10 D_{2} p^{Z_{1}^{\prime}}+5 p^{2 Z_{1}^{\prime}}=-1 .
$$


It implies that

$$
\left(4 D_{2}-5 p^{Z_{1}^{\prime}}\right)^{2}-5 p^{2 Z_{1}^{\prime}}=-4 .
$$

For any nonnegative integer $m$, let $F_{m}$ and $L_{m}$ denote the $m$ th Fibonacci number and the $m$ th Lucas number, respectively. Notice that all solutions $(X, Y)$ of the equation

$$
X^{2}-5 Y^{2}=-4, \quad X, Y \in \mathbb{N}, \quad \operatorname{gcd}(X, Y)=1
$$

are given by $(X, Y)=\left(L_{6 l}, F_{6 l+1}\right)$ and $\left(L_{6 l+5}, F_{6 l+5}\right)$, where $l$ runs through all nonnegative integers. We see from $(60)$ that

$$
\left(D_{2}, p^{Z_{1}^{\prime}}\right)=\left(\frac{1}{2} L_{6 l}, F_{6 l+1}\right) \quad \text { or } \quad\left(\frac{1}{2} L_{6 l+6}, F_{6 l+5}\right) .
$$

On the other hand, by (53) and (57), we have

$p^{2 Z_{1}^{\prime}}<\left(\frac{2 p^{5 Z_{1}^{\prime}}}{D_{2}}\right)^{1 / 2}<\frac{\pi}{2 \arcsin \left(D_{2} / 2 p^{5 Z_{1}^{\prime}}\right)^{1 / 2}}<t_{2}^{\prime}<3+2563.43\left(1+\frac{10.98 \pi}{\log p^{Z_{1}^{\prime} / 2}}\right)$,

whence we calculate that $p^{Z_{1}^{\prime}}<200$. Therefore, we find from (61) that $\left(D_{1}, D_{2}, p^{Z_{1}^{\prime}}\right)$ $=(1,9,5),(17,9,13)$ and $(17,161,89)$. Further, by $(54)$, we get

$$
\left|\arcsin \left(\frac{D_{2}}{2 p^{Z_{1}^{\prime}}}\right)^{1 / 2}-\frac{k \pi}{t_{2}^{\prime}}\right|<\frac{\pi}{2 t_{2}^{\prime} p^{Z_{1}^{\prime}\left(t_{2}^{\prime}-1\right) / 2}}
$$

for some positive integers $k$ with $k \leq\left(t_{2}^{\prime}-1\right) / 2$. However, if $t_{2}^{\prime}$ satisfies (62), then (63) is impossible.

If $t_{2}^{\prime}=7$, then from (52) we get

$$
\left(2 p^{Z_{1}^{\prime}}-2 D_{2}\right)^{3}+\left(2 p^{Z_{1}^{\prime}}-2 D_{2}\right)^{2} p^{Z_{1}^{\prime}}-2\left(2 p^{Z_{1}^{\prime}}-2 D_{2}\right) p^{2 Z_{1}^{\prime}}-p^{3 Z_{1}^{\prime}}=(-1)^{\left(p^{Z_{1}^{\prime}}+1\right) 2} .
$$

By Lemma 7 , we obtain from $(64)$ that $\left(D_{1}, D_{2}, p^{Z_{1}^{\prime}}\right)=(7,11,9)$. For this case, by (53), (54) and (57), we get

$$
729<t_{2}^{\prime}<83099
$$

and

$$
\left|\arcsin \left(\frac{11}{18}\right)^{1 / 2}-\frac{k \pi}{t_{2}^{\prime}}\right|<\frac{\pi}{2 \cdot 3^{t_{2}^{\prime}-1} t_{2}^{\prime}}
$$

for some positive integers $k$ with $k \leq\left(t_{2}^{\prime}-1\right) / 2$. However, (66) is impossible if $t_{2}^{\prime}$ satisfies (65).

By (53) and (57), if $t_{1}^{\prime}>7$, then $t_{1}^{\prime} \geq 11$ and $p^{Z_{1}^{\prime}} \leq 9$. Except for the already considered cases, we have $\left(D_{1}, D_{2}, p^{Z_{1}^{\prime}}\right)=(5,1,3),(1,9,5),(9,1,5),(1,13,7)(5.9,7)$, $(9,5,7),(11,3,7),(13,1,7),(1,17,9),(11,7,9),(13,5,9)$ or $(17,1,9)$. For these cases, (52) is false when $t_{1}^{\prime}=11,13,17$ and 19 . So we have $t_{1}^{\prime} \geq 23$. Then, by (53) and (57) again, we get

$$
\begin{aligned}
177147 \leq p^{11 Z_{1}^{\prime}} \leq p^{Z_{1}^{\prime}\left(t_{1}^{\prime}-1\right) / 2}<\frac{\pi}{2 \arcsin \left(D_{2} / 2 p^{s_{1}^{\prime}}\right)^{1 / 2}}<t_{2}^{\prime}<3+2563.43 \\
\times\left(1+\frac{10.98 \pi}{\log 3^{1 / 2}}\right)<153544
\end{aligned}
$$

a contradiction. The theorem is proved. 


\section{Proof of the Corollary}

Lemma 12 ([11]). The equation

$$
z^{2}+4=y^{n}, \quad x, y, n \in \mathbb{N}, \quad 2 \nmid y, \quad n>3,
$$

has no solution $(z, y, n)$.

Proof of Corollary. Let $(x, y, m, n)$ be a solution of (2) such that $m=3$ and $y$ is a prime power. Then we have

$$
(y-1)(2 x+1)^{2}+(3 y+1)=4 y^{n}, \quad n>3 .
$$

If $y=2$, then from (67) we get

$$
(2 x+1)^{2}+7=2^{n+2}, \quad n>3 .
$$

By [12], we find from $(68)$ that $(x, y, m, n)=(5,2,3,5)$ and $(90,2,3,13)$.

If $y=2^{k}$, where $k$ is a positive integer with $k>1$, then

$$
\left(2^{k}-1\right)(2 x+1)^{2}+\left(3 \cdot 2^{k}+1\right)=2^{k n+2}, \quad n>3 .
$$

Let $D_{1}=2^{k}-1, D_{2}=3 \cdot 2^{k}+1$ and $p=2$. We see from (69) that $(r, s)=$ $(2 x+1, k n+2)$ is a solution of (3) with $s>3 k+2$. However, by [8], then (3) has exactly two solutions $(r, s)=(1, k+2)$ and $\left(2^{k+1}+1,3 k+2\right)$. Therefore, $(69)$ is impossible.

If $2 \nmid y$, then $y=p^{k}$, where $p$ is an odd prime and $k$ is a positive integer.

If $y=5$, then from (67) we get

$$
(2 x+1)^{2}+4=5^{n}, \quad n>3 .
$$

By Lemma 12, (70) is impossible.

If $y \equiv 1(\bmod 4)$ and $y>5$, then we have

$$
\left(\frac{p^{k}-1}{4}\right)(2 x+1)^{2}+\left(\frac{3 p^{k}+1}{4}\right)=p^{k n}, \quad n>3 .
$$

Let $D_{1}=\left(p^{k}-1\right) / 4$ and $D_{2}=\left(3 p^{k}+1\right) / 4$. We see from $(71)$ that $(r, s)=(2 x+1, k n)$ is a solution of (3) with $s>3 k$. Notice that (3) has two solutions $(r, s)=(1, k)$ and $\left(2 p^{k}+1,3 k\right)$ in this case. Therefore, by Theorem $1,(71)$ is impossible.

Similarly, if $y \equiv 3(\bmod 4)$, then we have

$$
\left(\frac{p^{k}-1}{2}\right)(2 x+1)^{2}+\left(\frac{3 p^{k}+1}{2}\right)=2 p^{k n}, \quad n>3 .
$$

Let $D_{1}=\left(p^{k}-1\right) / 2$ and $D_{2}=\left(3 p^{k}+1\right) / 2$. Since (4) has two solutions $\left(r^{\prime}, s^{\prime}\right)=$ $(1, k)$ and $\left(2 p^{k}+1,3 k\right)$ in this case, by Theorem $2,(72)$ is impossible. Now, the corollary is proved.

\section{ACKNowledgements}

The author is grateful to the referees for their valuable suggestions. 


\section{REFERENCES}

[1] R. Apéry, Sur une équation diophantienne, C. R. Acad. Sci. Paris Sér. A 251 (1960), 12631264. MR 22:10951

[2] R. Apéry, Sur une équation diophantienne, C. R. Acad. Sci. Paris Sér. A 251 (1960), 14511452. MR 22:10950

[3] V. I. Baulin, On an indeterminate equation of the third degree with least positive discriminant, Tul'sk Gos. Ped. Inst. Učen. Zap. Fiz. Mat. Nauk Vyp. 7 (1960), 138-170. (in Russian) MR 33:7298

[4] R. Goormaghtigh, L'intermédiaire des Mathématiciens, 24 (1917), 88.

[5] R. K. Guy, Unsolved Problems in Number Theory, Springer-Verlag, New York, 1981. MR 83k:10002

[6] M. Laurent, M. Mignotte and Y. Nesterenko, Formes linéaires en deux logarithmes et déterminants d'interpolation, J. Number Theory, 55 (1995), 285-321. MR 96h:11073

[7] M.-H. Le, The divisibility of the class number for a class of imaginary quadratic fields, Kexue Tongbao, 32 (1987), 724-727. (in Chinese) CMP 20:01

[8] M.-H. Le, The diophantine equation $D_{1} x^{2}+D_{2}=2^{n+2}$, Acta Arith., 64 (1993), 29-41. MR 94:11030

[9] M.-H. Le, A note on the generalized Ramanujan-Nagell equation, J. Number Theory, 50 (1995), 193-201. MR 96f:11051

[10] R. Lidl and H. Niederreiter, Finite Fields, Addison-Wesley, Reading, MA, 1983. MR 86c: 11106

[11] T. Nagell, Contributions to the theory of a category of diophantine equations of the second degree with two unknowns, Nova Acta Soc. Sci. Upsal., (4) 16, no. 2, 38pp. (1955). MR $17: 13 b$

[12] T. Nagell, The diophantine equation $x^{2}+7=2^{n}$, Arkiv. Mat., 4 (1960), 185-187.

[13] R. Ratat, L'Intermediaire des Mathématiciens, 23 (1916), 150.

[14] T. N. Shorey, Some exponential diophantine equations, in Number Theory and Related Topics, pp. 217-229, Bombay, 1989. MR 98d:11038

Department of Mathematics, Zhanjiang Teachers College, Postal Code 524048, ZhanJiang, Guangdong, P. R. China 